\title{
Lifetime of Heavy Composite Systems Formed by Fusion between Heavy Nuclei
}

\author{
Toshiki Maruyama, ${ }^{*, a}$ Aldo Bonasera, ${ }^{\mathrm{b}}$ Massimo Papa, ${ }^{\mathrm{b}, \mathrm{c}}$ and Satoshi Chiba ${ }^{\mathrm{a}}$ \\ a Japan Atomic Energy Research Institute, Tokai, Ibaraki 319-1195, Japan \\ ${ }^{\mathrm{b}}$ Istituto Nazionale di Fisica Nucleare Laboratorio Nazionale del Sud, Via S. Sofia 44, Catania 95123, \\ Italy \\ ${ }^{\mathrm{c}}$ Istituto Nazionale di Fisica Nucleare Sezione di Catania, Corso Italia 57, Catania 95129, Italy
}

\section{Received: December 19, 2001; In Final Form: December 19, 2001}

\begin{abstract}
We investigate the formation and the decay of heavy systems which are above the fission barrier. By using a microscopic simulation of constrained molecular dynamics (CoMD) on Au + Au collision, we observe composite states stay for very long time before decaying by fission.
\end{abstract}

\section{Introduction}

Collisions between heavy nuclei at relatively low energy region have attracted strong interests of heavy-ion physicists for 3 different reasons, namely the creation of super heavy elements (SHEs), fission dynamics of very heavy systems, and creation of electron-positron pair due to the strong Coulomb field of the composite heavy nuclei as a verification of the QED process. In these processes, the lifetime of the composite system, created by the fusion of the projectile and the target, which decays eventually by fission is the key issue to understand the underlying reaction mechanisms and to estimate the probability of occurrence of these processes.

SHEs are produced in two ways: one is "cold fusion" which is complete fusion below the classical barrier, and the other is "hot fusion" which allows several neutrons to be emitted. Even though the name is "hot", such reactions are still at very low energy near the barrier and the total mass number is very close to the aimed one. As far as the formation of SHE is concerned, the "fusion" of very heavy nuclei where the fission barrier no more exists is found to be ineffective. ${ }^{1,2}$

In the study of fission dynamics of heavy systems including the spontaneous fission and the fusion-fission of heavy composite, the competition of neutron emission between the fission and the fission delay have been discussed intensively. However almost all the discussion are done for mass regions where the classical fission barrier exists.

Sometime ago many physicists paid attention to the low energy collision of very heavy nuclei in regard to the spontaneous positron emission from strong electric fields. ${ }^{3}$ If a molecule state of, say, $U$ and $U$ is formed and stays sufficiently long time, the binding energy of an electron can exceed the electron mass and might create electron-positron pair by a static QED process. Unfortunately no clear evidence of static positron creation was observed below Coulomb energy region. They have pointed out ${ }^{4}$ the importance of nuclear reaction which causes the time delay of separation of two nuclei. Although there increases the background component of positrons from nuclear excitation, which in this case is not interested in, the electron-positron from the static QED process is also expected to increase. However, the reaction mechanism of very heavy nuclei has not been discussed by fully dynamical models.

In this paper we discuss the possibility of molecule-like states of heavy nuclei and the time scale of very heavy composite system formed by the fusion-fission or deep inelastic processes. To investigate these problems theoretically we use a recently developed constrained molecular dynamics (CoMD) model. ${ }^{5}$ This model has been proposed to include the Fermionic nature of constituent nucleons by a constraint that the phase space distribution should always satisfy the condition $f \leq 1$.

*Corresponding author. E-mail: maru@ hadron02.tokai.jaeri.go.jp.
In this paper we apply CoMD to the investigation of ${ }^{197} \mathrm{Au}+{ }^{197} \mathrm{Au}$ collisions at low energies where fusion-fission or deep-inelastic process may occur. In the following we give a brief review of the model. ${ }^{5}$

\section{Constrained Molecular Dynamics Model}

The CoMD model mainly consists of two parts: classical equation of motion of many-body system, and stochastic process which includes constraint of Pauli principle and the twobody collisions. We write the distribution function of the system as a sum of one-body distribution function neglecting the antisymmetrization

$$
\begin{aligned}
f(\mathbf{r}, \mathbf{p}) & =\sum_{i} f_{i}(\mathbf{r}, \mathbf{p}), \\
f_{i}(\mathbf{r}, \mathbf{p}) & =\frac{1}{\left(2 \pi \sigma_{r} \sigma_{p}\right)^{3}} \cdot \exp \left[-\frac{\left(\mathbf{r}-\mathbf{R}_{i}\right)^{2}}{2 \sigma_{r}^{2}}-\frac{\left(\mathbf{p}-\mathbf{P}_{i}\right)^{2}}{2 \sigma_{p}^{2}}\right] .
\end{aligned}
$$

The equation of motion of $\mathbf{R}_{i}$ and $\mathbf{P}_{i}$ are derived using the timedependent variational principle which gives:

$$
\dot{\mathbf{R}}_{i}=\frac{\partial H}{\partial \mathbf{P}_{i}}, \quad \dot{\mathbf{P}}_{i}=-\frac{\partial H}{\partial \mathbf{R}_{i}}
$$

In our approach the total energy $H$ for $A$ particles with mass $m$ consists of the kinetic energy and the effective interactions:

$$
H=\sum_{i} \frac{\mathbf{P}_{i}^{2}}{2 m}+A \frac{3 \sigma_{p}^{2}}{2 m}+V .
$$

The second term arises from the Gaussian width in $p$-space. However in the following considerations we omit such a constant term.

The effective interaction $V$ we adopt is written as

$$
V=V^{\text {vol }}+V^{(3)}+V^{\text {sym }}+V^{\text {surf }}+V^{\text {coul }}
$$

By defining the superimposition integral $\rho_{i j}$ as:

$$
\begin{aligned}
\rho_{i j} & \equiv \int d^{3} r_{i} d^{3} r_{j} \rho_{i}\left(\mathbf{r}_{i}\right) \rho_{j}\left(\mathbf{r}_{j}\right) \delta\left(\mathbf{r}_{i}-\mathbf{r}_{j}\right) \\
\rho_{i} & \equiv \int d^{3} p f_{i}(\mathbf{r}, \mathbf{p})
\end{aligned}
$$

the terms in eq 5 can be written as:

$$
\begin{aligned}
V^{\mathrm{vol}} & =\frac{t_{0}}{2 \rho_{0}} \sum_{i, j \neq i} \rho_{i j}, \\
V^{(3)} & =\frac{t_{3}}{(\mu+1)\left(\rho_{0}\right)^{\mu}} \sum_{i, j \neq i} \rho_{i j}^{\mu}, \\
V^{\mathrm{sym}} & =\frac{a_{\mathrm{sym}}}{2 \rho_{0}} \sum_{i, j \neq i}\left[2 \delta_{\tau_{i}, \tau_{j}}-1\right] \rho_{i j},
\end{aligned}
$$




$$
\begin{aligned}
V^{\text {surf }} & =\frac{C_{s}}{2 \rho_{0}} \sum_{i, j \neq i} \nabla_{\mathbf{R}_{i}}^{2}\left(\rho_{i j}\right), \\
V^{\text {Coul }} & =\frac{1}{2} \sum_{\substack{i, j \neq i \\
(i, j \in \text { protons })}} \frac{e^{2}}{\left|\mathbf{R}_{i}-\mathbf{R}_{j}\right|} \operatorname{erf}\left(\frac{\left|\mathbf{R}_{i}-\mathbf{R}_{j}\right|}{2 \sigma_{r}^{2}}\right) .
\end{aligned}
$$

In the above relations the coordinate $\tau_{i}$ represents the nucleon isospin degree of freedom.

In this paper we use the parameter set for wave packet width and effective interactions: $\sigma_{r}=1.15 \mathrm{fm}, \sigma_{p} / \hbar=0.4748 \mathrm{fm}^{-1}$, $t_{0}=-301.1 \mathrm{MeV}, t_{3}=242 \mathrm{MeV}, \mu=7 / 6, a_{\mathrm{sym}}=26.4 \mathrm{MeV}$, $C_{s}=-0.165 \mathrm{MeV} \mathrm{fm}^{2}$, and $\rho_{0}=0.165 \mathrm{fm}^{-3}$, which reproduces reasonably well the fusion cross section of $\mathrm{Ca}+\mathrm{Ca}$ reactions, while in Reference 5 we have used another set of parameters.

The Pauli principle is taken into account in two ways: one is the Pauli blocking of the final state of two-body collision and the other is the constraint which brings into the system the Fermi motion in a stochastic way. The starting point of the constraint is the requirement:

$$
\begin{aligned}
& \bar{f}_{i} \leq 1 \quad(\text { for all } i) \\
& \bar{f}_{i} \equiv \sum_{j} \delta_{\tau_{i}, \tau_{j}} \delta_{s_{i}, s_{j}} \int_{h^{3}} f_{j}(\mathbf{r}, \mathbf{p}) d^{3} r d^{3} p
\end{aligned}
$$

where $s_{i}$ is the spin coordinate of the nucleon $i$. The integral is performed in a hypercube of volume $h^{3}$ in the phase-space centered around the point $\left(\mathbf{R}_{i}, \mathbf{P}_{i}\right)$ with size $\sqrt{\frac{2 \pi \hbar}{\sigma_{r} \sigma_{p}}} \sigma_{r}$ and $\sqrt{\frac{2 \pi \hbar}{\sigma_{r} \sigma_{p}}} \sigma_{p}$ in the $r$ and $p$ spaces respectively.

At each time step and for each particle $i$ the phase space occupation $\bar{f}_{i}$ is checked. If $\bar{f}_{i}$ has a value greater than 1 an ensemble $K_{i}$ of nearest particles (including the particle $i$ ) is determined within the distances $3 \sigma_{r}$ and $3 \sigma_{p}$ in the phase space. Then we change randomly the momenta of the particles belonging to the ensemble $K_{i}$ in such a way that for the newly generated sample the total momentum and the total kinetic energy is conserved ("many-body elastic scattering"). The new sample is accepted only if it reduces the phase space occupation $\bar{f}_{i}$ (Ref. 5).

To handle the Pauli-blocking in the collision term is straightforward from the constraint. In fact for each NN collision we evaluate the occupation probability after the elastic scattering. If such functions are both less than 1 the collision is accepted, rejected otherwise. We note that for the results discussed here and especially at the lowest energies the collision term is of little importance.

\section{Collision of Au + Au System}

To simulate the collision of two ${ }^{197}$ Au nuclei, we prepare the ground state by applying the frictional cooling method together with the constraint of CoMD. The ground states we obtain have binding energy of $8.4 \mathrm{MeV} /$ nucleon and the root mean square

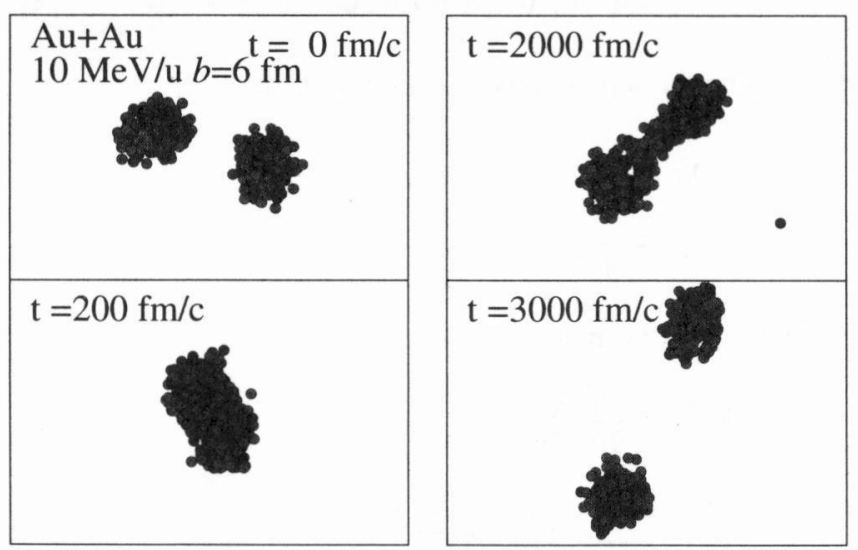

Figure 1. Snapshot of ${ }^{197} \mathrm{Au}+{ }^{197} \mathrm{Au}$ at $E_{\mathrm{lab}}=10 \mathrm{MeV} /$ nucleon $b=6 \mathrm{fm}$. The time indicated in each panel is not from the contact of two nuclei but indicates only that of the simulation. radius of $5.34 \mathrm{fm}$. They are rather stable for $1000 \mathrm{fm} / c$. For instance our ${ }^{197} \mathrm{Au}$ ground states evaporates 3.1 nucleons during $1000 \mathrm{fm} / c$. The collision events are performed for impact parameter $b$ of 0 and $6 \mathrm{fm}$ for incident energy in laboratory system of $E_{\text {lab }}=5 \sim 35 \mathrm{MeV} /$ nucleon.

Figure 1 shows a typical event of CoMD calculation with incident energy $E_{\text {lab }}=10 \mathrm{MeV} /$ nucleon with impact parameter $b=6 \mathrm{fm}$. The two nuclei form a quite deformed compound system, they keep such a deformation almost $2500 \mathrm{fm} / c$ and finally fission takes place. The system does not show much rotation since the angular momentum per nucleon is not so large and the elongated shape makes the moment of inertia larger than that in the initial stage. Therefore the reaction mechanism we are observing here may be in-between the deep inelastic and molecular resonance.

There are many observables which distinguish the reaction mechanism. The largest fragment mass is one of such welldefined observables which can easily be measured experimentally. Figure 2 shows the time dependences of the largest cluster mass for the impact parameters $b=0$ and $6 \mathrm{fm}$ calculated by CoMD and QMD. In CoMD calculations we see at the beginning the largest cluster mass $A_{\max }=197$ which corresponds to projectile and target mass number. Within about $50 \mathrm{fm} / c, A_{\max }$ becomes 394 except for the incident energy $E_{\text {lab }}=5 \mathrm{MeV}$ which is below the barrier where two nuclei never contact. At incident energies above the barrier, the formed large system will decay into smaller fragments by different modes according to the energy and angular momentum. At higher incident energies ( $E_{\text {lab }} \geq 30 \mathrm{MeV} /$ nucleon) the largest cluster mass changes suddenly at the early stage and continuously decreases in time. This indicates multifragmentation for head-on collisions and deep inelastic reaction for peripheral collisions followed by the emission of nucleons and small fragments. At lower incident energies ( $E_{\text {lab }} \leq 20 \mathrm{MeV} /$ nucleon) there is a sudden change of the largest cluster mass at very late time, which indicates a fission of the system. One should note that in our calculation of $\mathrm{Au}+\mathrm{Au}$ system there is almost no event where the system decays only by emitting particles or light fragments, i.e., pure incomplete fusion. The instability due to the Coulomb repulsion plays the major role in the decay process.

Here we should note that the plotted largest fragment mass are obtained by only one event for each incident energy and impact parameter. Therefore the fission time includes large amount of statistical error. In Figure 2(b) the same quantity as Figure 2(a) is displayed for QMD calculation. These QMD calculations are based on the same code as CoMD switching off the procedure for the constraint. The difference between the CoMD and the QMD is clear and dramatic. At low energy collisions there are no fission process and the system decays only by emitting nucleons and light fragments. At higher energies there is some sudden

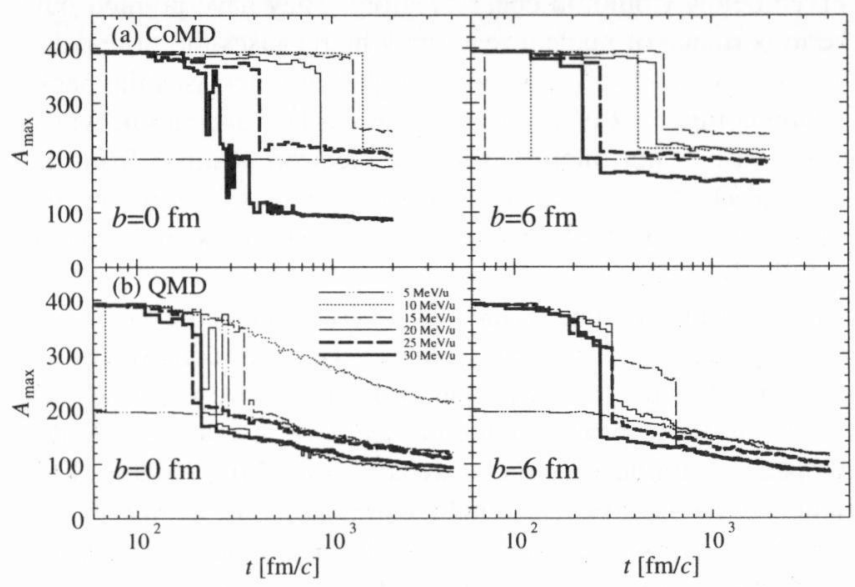

Figure 2. The time-dependence of the largest fragment mass $A_{\max }$ From the top (a) CoMD and (b) QMD. The left panels show cases of head-on collision and the right $b=6 \mathrm{fm}$. 
change of the largest fragment mass even in QMD calculation. This is not a fission but passing through for head-on collisions or deep inelastic process for peripheral collisions.

\section{Lifetime of Produced Composite Systems}

Assuming a very simple form of the time-dependent fission width $\Gamma(t)=\Gamma_{\mathrm{f}} \theta\left(t-T_{\mathrm{d}}\right)$, the averaged fission time $T_{\text {fiss }}$ can be obtained by the survival probability of the compound system against two-body process $P_{\text {surv }}$ as

$$
\begin{aligned}
P_{\text {surv }} & =\exp \left[-\left(t-T_{\mathrm{d}}\right) \Gamma_{\mathrm{f}} / \hbar\right] \\
T_{\text {fiss }} & \equiv T_{\mathrm{d}}+\hbar / \Gamma_{\mathrm{f}}
\end{aligned}
$$

where $T_{\mathrm{d}}$ is the delay time and $\Gamma_{\mathrm{f}}$ is the "fission width" after the delay time. The probability $P_{\text {surv }}(t)$ is obtained directly by the simulation. This fitting can apply well only for fission-like process in our calculation. Figure 3 shows the survival probability of a large fragment with $A>350$ which decays by fission-like mode or emitting large fragments $(A>30)$. The histograms are directly obtained by the simulation and the curves are fitting by eq 15,16 . For all the calculations the fitting works well, particularly the effect of delay time. The assumption of constant fission width after the delay time, on the other hand, is not completely supported because of poor statistics and still existing dynamical effects. One should note that the fitting by eq 15,16 is just to extract the "fission" time of the super heavy composite. Especially the time scale of QMD results is obviously different from that of fission process.

The extracted fission time $T_{\text {fiss }}$ are plotted in Figure 4 . With effective interaction used in Reference 5 the calculated fission time is about an order longer than this calculation. However in both cases of CoMD calculations the longest lifetime of heavy composite is found at $E_{\mathrm{lab}}=10 \mathrm{MeV} /$ nucleon.

For lower incident energies (just above the Coulomb barrier) the system might not form a fully thermalized single composite but might be quasi separated in the phase space, which makes the system split easily. For higher energies, the fully thermalized system needs some fluctuations to reseparate even though there

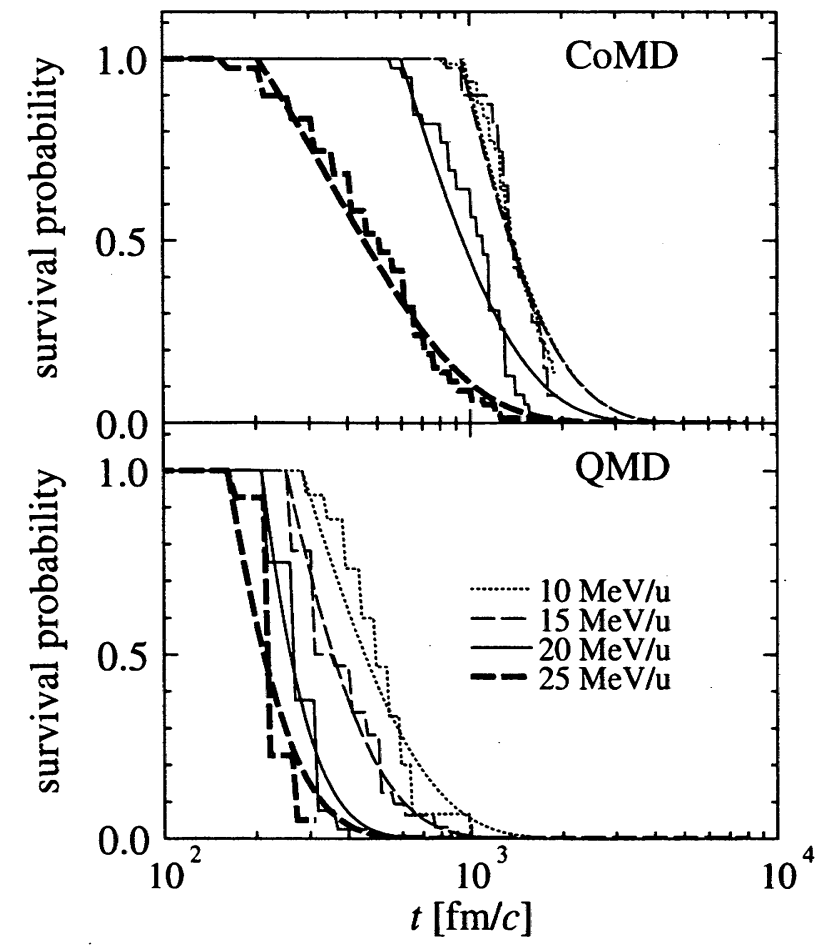

Figure 3. The survival probability of large fragments $(A>350)$ which decay by fission-like mode. The abscissa indicates the time after the contact of two nuclei. From the top the results are obtained for head-on collisions by CoMD and QMD. The histograms indicate results from simulations and smooth curves are the fits by eq 15,16 .

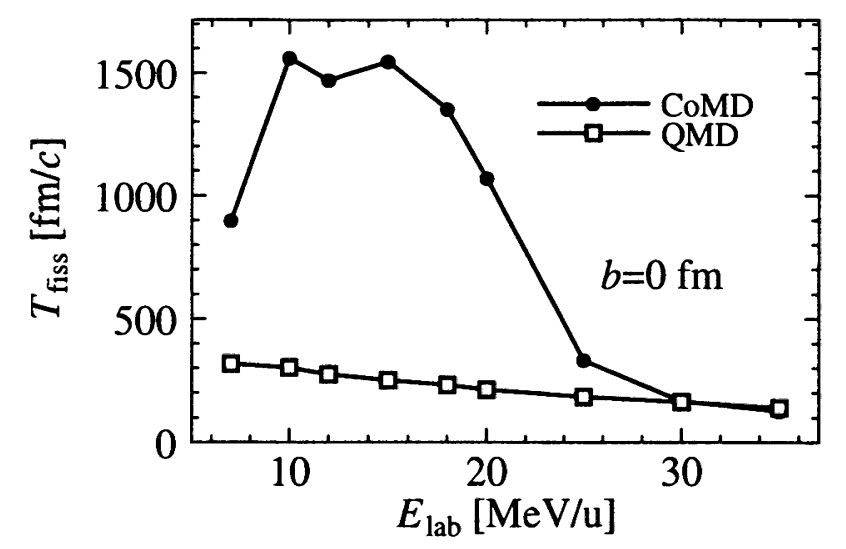

Figure 4. The "fission" lifetime of composite system for CoMD and QMD calculation.

is no classical barrier for fission. Therefore the fission time gets shorter with increase of the incident energy.

In QMD calculation what is in marked contrast to the CoMD calculation is that the "fission time" has no maximum energy and shows monotonic decrease. This is due to the lack of the Pauli principle which suppresses two nuclei from overlapping at very low energies (above the Coulomb barrier).

For peripheral collision $(b=6 \mathrm{fm})$, the lifetime of very heavy composite is shorter than the head-on collisions. But the incident-energy dependence is very similar to the $b=0 \mathrm{fm}$ cases. Though the mechanism is much more dynamical, eq 15,16 fit well again.

Nevertheless, the super heavy composite system formed by the head-on collision of $A u+A u$ may survive rather long time of $10^{3} \sim 10^{4} \mathrm{fm} / \mathrm{c}$.

An interesting aspect of the long-lived very heavy system is, as mentioned before, the spontaneous positron-electron production from the strong electric field as a static QED process. The total charge of $\mathrm{Au}+\mathrm{Au}$ system may be still smaller than the necessary charge $(Z \sim 170)$ for this process. However, the nuclear reaction of, e.g. $U+U$ system, should be qualitatively the same as what we observe in $\mathrm{Au}+\mathrm{Au}$ system. Although the background positrons should be larger, one can get longest lifetime

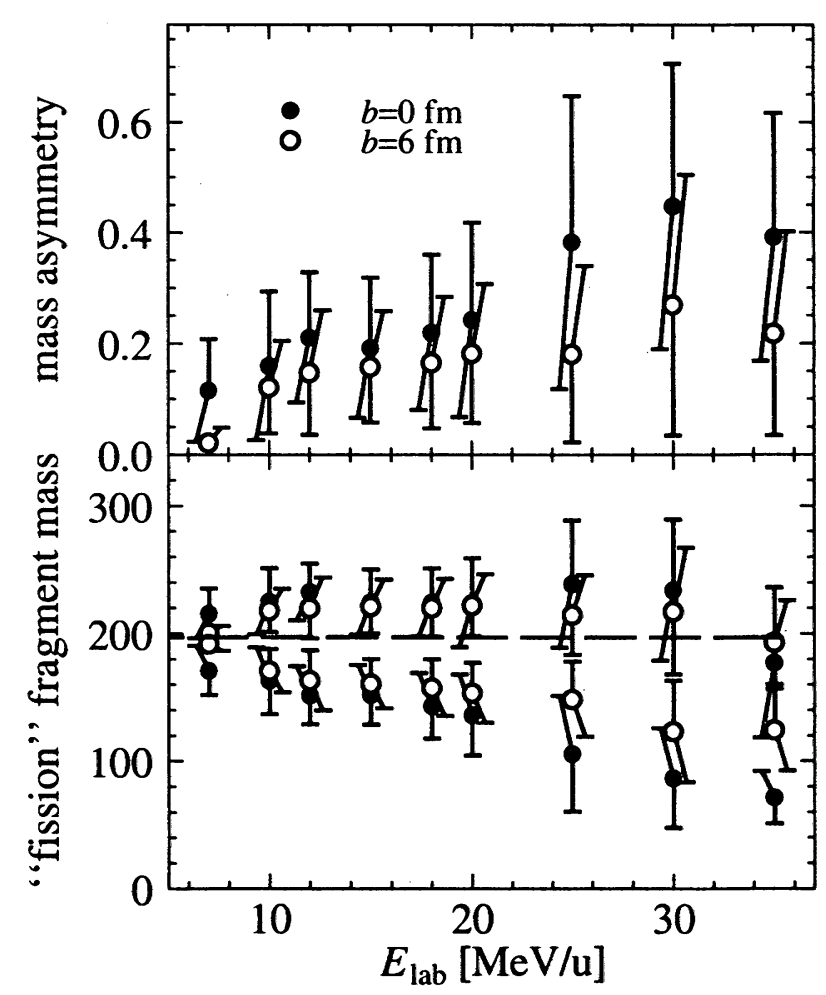

Figure 5. The mass asymmetry of the fission fragments and the fragment mass. The dashed line indicates the initial mass of projectile and target nuclei. Error bars indicate statistical standard deviation. 
of strong electric field (stronger than the case of Rutherford or molecular trajectory) around $E_{\mathrm{lab}}=10 \mathrm{MeV} /$ nucleon at some impact parameter and the production of positrons from the static QED process should be largest around that energy.

\section{Asymmetric Fission}

As mentioned above, production of SHE is one of the most important subject in the heavy-ion collision problem. Besides cold- and hot-fusion, mass transfer in collision of very heavy nuclei was tried before. One could produce, e.g. up to Fm $(Z=100)$ in $U+U$ system, or $M d(Z=101)$ in $U+C m$ system, by such a mechanism. ${ }^{1,2}$ The incident energy, however, was very close to the Coulomb barrier and the reaction was rather gentle with the transfer of $\sim 20$ nucleons. In our CoMD calculation for $E_{\text {lab }} \geq 7 \mathrm{MeV} /$ nucleon, the reaction mechanism is more violent and there happens the transfer of much more nucleons though the mass loss from the system is also large. In Figure 5 plotted is the mass-asymmetry $\left(A_{1}-A_{2}\right) /\left(A_{1}+A_{2}\right)$ of the fission process in CoMD calculation and the fission fragment mass $A_{1}$ and $A_{2}\left(<A_{1}\right)$. The mass-asymmetry increases with the incident energy. For $b=0$ the asymmetry amounts to about 0.1 at $E_{\text {lab }}=7 \mathrm{MeV} /$ nucleon and almost 0.4 at $30 \mathrm{MeV} /$ nucleon. As a result, the largest fission fragment can be much larger than the initial projectile and target nuclei as shown at the lower panel of the figure. Of course we should consider the thermal mass loss and subsequent fission due to the excitation of fragments. However, such kind of fusion-fission mechanism at around $10 \mathrm{MeV} /$ nucleon should be taken into account for the SHE production. The new $4 \pi$ detectors can accumulate lots of statistics plus they can make coincidence studies to see if the fragments come from fission.

\section{Summary}

In summary, we have discussed the formation and decay of super heavy composite in the Au + Au collisions. The CoMD calculation which takes into account the Fermionic nature of the nucleon many-body system can describe well the low-energy dynamics including fusion, fission, deep inelastic, emission of nucleons and small fragments, and multifragmentation. Although there are still some ambiguities on the effective interaction, the lifetime of super heavy composite is found to be rather long up to $10^{3} \sim 10^{4} \mathrm{fm} / c$. Some experimental explorations such as detection of $e^{+} e^{-}$formation at around $10 \mathrm{MeV} /$ nucleon and measurement of the energy averaged angular distribution and/or excitation function for binary processes are encouraged.

\section{References}

(1) H. Gäggeler, M. Schädel, W. Brüchle, J. V. Kratz, K. Sümmerer, G. Wirth, N. Trautmann, P. Peuser, G. Tittel, R. Stakemann, G. Herrmann, E. K. Hulet, R. W. Lougheed, J. M. Nitschke, R. L. Hahn, and R. L. Ferguson, Proceedings of the 4th International Conference on Nuclei Far From Stability, Helsinger, 1981, edited by L. O. Skolen, CERN 8109, 763 (1981).

(2) G. Herrmann, Proceedings of the 4th International Conference on Nuclei Far From Stability, Helsinger, 1981, edited by L. O. Skolen, CERN 81-09, 772 (1981).

(3) Quantum Electrodynamics of Strong Fields, edited by W. Greiner (Plenum Press, New York and London, 1983); R. Bär, A. Balanda, J. Baumann, W. Berg, K. Bethge, H. Bokemeyer, H. Folger, O. Fröhlich, R. Ganz, O. Hartung, M. Samek, P. Salabura, W. Schön, D. Schwalm, K. Stiebing, P. Thee, E. Berdermann, F. Heine, S. Heinz, O. Joeres, P. Kienle, I. Koenig, W. Koenig, C. Kozhuharov, U. Leinberger, A. Schröter, and H. Tsertos, Nucl. Phys. A 583, 237 (1995); I. Ahmad, S. M. Austin, B. B. Back, D. Bazin, R. R. Betts, F. P. Calaprice, K. C. Chan, A. Chishti, P. Chowdhury, R. W. Dunford, J. D. Fox, S. J. Freedman, M. Freer, S. B. Gazes, J. S. Greenberg, A. L. Hallin, T. Happ, J. Last, N. Kaloskamis, E. Kashy, W. Kutschera, C. J. Lister, M. Liu, M. R. Maier, D. Mercer, A. Perera, M. D. Rhein, D. E. Roa, J. P. Schiffer, T. Trainor, P. Wilt, J. S. Winfield, M. Wolanski, F. L. H. Wolfs, A. H. Wuosmaa, G. $\mathrm{Xu}, \mathrm{A}$. Young, and J. E. Yurkon, Nucl. Phys. A 583, 247 (1995); A. Lépine-Szily, J. M. Casandjian, W. Mittig, A. C. C. Villari, R. Lichtenthäler Filho, G. Auger, L. Bianchi, R. Beunard, M. E. Brandan, J. L. Ciffre, A. Cunsolo, A. Foti, L. Gaudard, A. Menchaca-Rocha, N. A. Orr, E. Plagnol, Y. Schutz, R. H. Siemssen, and J. P. Wieleczko, Nucl. Phys. A 583, 263 (1995).

(4).J. Reinhardt, in Quantum Electrodynamics of Strong Fields, edited by W. Greiner (Plenum Press, New York and London, 1983).

(5) M. Papa, T. Maruyama, and A. Bonasera, Phys. Rev. C 64, 024612 (2001). 